\title{
Percepção Materna de Movimentos Fetais como Método de Avaliação da Vitalidade Fetal em Gestantes Diabéticas
}

\author{
Maternal Perception of Fetal Movements as a Method \\ to Evaluate Fetal Condition in Diabetic Women
}

Belmiro Gonçalves Pereira ${ }^{1}$, Aníbal Faúndes ${ }^{1,2}$, Mary Angela Parpinelli ${ }^{1}$, José Carlos Gama da Silva ${ }^{1}$

\section{RESUMO}

Objetivo: avaliar a acurácia da percepção materna dos movimentos fetais (PMMF) na predição de alguns resultados perinatais em gestantes diabéticas.

Métodos: análise retrospectiva de 209 gestantes diabéticas atendidas no Centro de Atenção Integral à Saúde da Mulher (CAISM), entre junho de 1988 e maio de 1996, que tinham registro de PMMF dentro dos três dias anteriores ao parto, monitorização eletrônica da freqüência cardiaca fetal intraparto, idade gestacional $\geq 30$ semanas, avaliação do recém-nascido (RN) $e$ diagnóstico neonatal. O teste de PMMF foi considerado normal quando a mulher detectou um minimo de 7 movimentos em 60 minutos.

Resultados: a sensibilidade do teste foi de 23 e 29\% para Apgar $<7$ aos 5 minutos e sofrimento fetal intraparto (SFI), respectivamente, e próximo a $50 \%$ para anóxia neonatal (45,5\%). A especificidade foi em torno de $95 \%$ para os três indicadores e o valor preditivo negativo (VPN) foi de 80\% para SFI, mas de 98 e 97\% para Apgar $<7$ aos 5 minutos e anóxia neonatal, respectivamente.

Conclusões: a PMMF é um teste de grande utilidade para rastreamento das gestantes diabéticas que precisam de avaliação fetal por técnicas mais complexas, dado o alto VPN, que indica a capacidade de separar os casos em que a condição do feto é normal.

PALAVRAS-CHAVE: Diabetes na gestação. Sofrimento fetal. Mortalidade perinatal. Diabetes mellitus.

\section{Introdução}

Atualmente, a gestante diabética oportunamente diagnosticada consegue com freqüência um controle metabólico razoável durante a fase pré-gestacional e durante a gestação. Resta avaliar as causas de morbidade e mortalidade perinatal relacionadas à prematuridade e ao sofrimento fetal intraparto (SFI) que, como sabemos, pode ser uma das condições que justificam a interrupção prematura

\footnotetext{
${ }^{1}$ Departamento de Ginecologia e Obstetrícia, Faculdade de Ciências Médicas, Universidade Estadual de Campinas (UNICAMP), Campinas, SP.

${ }_{2}^{2}$ Centro de Pesquisa das Doenças Materno-Infantis de Campinas (CEMICAMP), Campinas, SP.

Correspondência:

Belmiro G. Pereira

Caixa Postal 6030

13091-970 - Campinas - SP
}

Auxilio financeiro, equipamentos e drogas: FCM/UNICAMP; CEMICAMP em gestações complicadas por diabetes ${ }^{1,2}$. Desta forma, a avaliação do bem-estar fetal passou a ser uma das prioridades nos principais centros de assistência às gestações de diabéticas.

A incidência de prematuros entre as gestantes portadoras de diabete é razoavelmente elevada: $17,4 \%$ nos EUA $^{3}$ e $20 \%$ na Suécia ${ }^{4}$. Na nossa casuística este valor foi muito semelhante $\left(17,7 \%^{5}\right)$, mas chama a atenção que a incidência de trabalho de parto prematuro foi de apenas 5,6\%. Explica-se tal diferença porque a maioria destes partos correspondeu à interrupção prematura da gravidez por indicação médica ${ }^{5}$.

Há duas principais indicações médicas para interrupção prematura de gestação em diabéticas: uma está relacionada ao descontrole metabólico; a outra, relacionada às condições de bem-estar fetal. Esta última representou $26 \%$ das indicações de cesárea, mas apenas $16 \%$ dos RN tiveram indices de Apgar menor que sete ao primeiro minuto de vida, numa casuística de 90 gestantes 
diabéticas atendidas no $\mathrm{CAISM}^{5}$. Outros autores nacionais relataram incidência de 30\% de depressão neonatal entre os filhos de mães com diabetes $^{6}$.

Os métodos de avaliação do bem-estar fetal são freqüentemente usados de forma indiscriminada em gestantes com diabete, hipertensão e outras patologias, e os resultados interpretados como se as doenças fossem idênticas.

Uma das formas mais conhecidas e mais antigas para se avaliar a vitalidade dos conceptos é a percepção da movimentação fetal pelo obstetra, mas principalmente pela gestante. No entanto, é um método que, de tão simples, vem sendo abandonado na prática de assistência pré-natal e substituido por novas tecnologias aparentemente mais eficazes, que utilizam aparelhos sofisticados e permitem registro documental, oferecendo ao médico a impressão de melhor controle. As gestantes são capazes de perceber $90 \%$ de todos os movimentos fetais, independentemente do grau de instrução, paridade, idade gestacional, obesidade e local de inserção placentária, quando comparados com avaliação ultra-sonográfica em tempo real $^{7,8}$.

A partir de 1986, na Disciplina de Obstetricia do DTG (FCM UNICAMP), passamos a realizar sistematicamente, nas gestações de risco, o registro da percepção materna dos movimentos fetais (PMMF) em períodos aleatórios do dia. Estes são registrados em formulário próprio, em que a gestante anota o horário de início e o de término quando atinge sete movimentos, se isso ocorrer antes de uma hora. Se neste tempo não ocorrerem os sete movimentos, a gestante é orientada a repeti-lo após uma refeição ${ }^{9}$.

A análise dessa experiência permitiu concluir que o teste oferece elevada especificidade e baixa porcentagem de falso-negativos, mas porcentagem relativamente alta de falso-positivos. Em outras palavras, os fetos de boa movimentação estavam em boas condições de vitalidade; já os de movimentos reduzidos não estavam, necessariamente, em más condições, devendo, outrossim, serem submetidos a testes mais sensiveis de avaliação fetal ${ }^{10}$.

Em nosso Serviço, recomendamos a avaliação da vitalidade fetal, nas diabéticas, a partir das 28 semanas pelo controle dos movimentos fetais realizado pela gestante. Este procedimento é acrescido da cardiotocografia (CTG) após a 30 a semana. A CTG é realizada a intervalos regulares, de acordo com a idade gestacional: entre 30-34 semanas, é realizada uma vez por semana; entre 34-36 semanas, duas vezes por semana; acima de 36 semanas, diariamente, até o dia do parto, que preferencialmente deve ser aguardado ao termo. A dopplerfluxometria e o perfil biofísico fetal são reservados às situações em que a CTG está alterada ${ }^{11}$. Consideramos de grande importância avaliar, com precisão, os limites de capacidade de diagnóstico de um método tão simples como a PMMF, que tem a vantagem de poder se realizar diariamente, e até mais de uma vez por dia, desde que se julgue necessário, a custo praticamente zero e com tecnologia acessivel a todos.

Pensamos que obter estas informações, até agora não-disponiveis, permitirá fazer recomendações muito melhor fundamentadas quanto aos procedimentos a seguir na avaliação dos fetos das gestantes diabéticas. Para cumprir este objetivo, revisamos a nossa experiência de oito anos, durante os quais acompanhamos 209 gestações de diabéticas que realizaram os registros de $\mathrm{PMMF}$.

\section{Pacientes e Métodos}

Foram avaliados os registros da PMMF em 209 gestações de diabéticas, atendidas no Pré-Natal Especializado (PNE), que tiverem parto no CAISM entre junho de 1988 e maio de 1996.

Foram incluidas todas as gestantes diabéticas que ingressaram no PNE. Estas tiveram o controle metabólico, avaliado por perfil glicêmico, na semana anterior ao parto. Foi realizado o controle da PMMF dentro dos três dias anteriores ao parto. O trabalho de parto foi monitorizado eletronicamente. Foi efetuada avaliação neonatal da idade gestacional somática- ${ }^{12}$ e aplicado o indice de Apgar no primeiro e quinto minutos de vida ${ }^{13,14}$. Nos casos de óbito fetal foi considerada a idade gestacional baseada no tempo de amenorréia. Também foram critérios de inclusão ter 30 semanas ou mais de idade gestacional e os dados da evolução obstétrica e neonatal anotados em prontuário.

Foram excluídas do estudo as gestantes que tiveram RN com malformações cardiacas, neurológicas ou outras grandes malformações, as que tiveram sinais de sofrimento fetal intraparto não-relacionado ao diabete (descolamento prematuro de placenta, prolapso de cordão umbilical) e aquelas com gestação múltipla.

$O$ teste de PMMF foi classificado como normal se ocorressem até sete movimentos em uma hora ou menos e como alterado se fossem registrados até seis movimentos no período de uma hora.

Foram utilizados como indicadores do estado do $\mathrm{RN}$ os índices de Apgar ao 1으 e 5o minutos de vida, classificados em duas categorias: de 0 a 6 e de 7 a 10 . A presença de SFI foi baseado na prova 
de Pose positiva, com desacelerações tardias em $30 \%$ ou mais das contrações uterinas ou bradicardia sustentada durante o trabalho de parto espontâneo ou induzido. Foi também utilizado o diagnóstico de anóxia neonatal, definida pela presença de alteração neurológica e classificada, qualitativamente, como presente ou ausente.

Além disso, foi analisada a presença ou não de hipertensão arterial materna. Por último, foram registradas uma série de variáveis que definem as características do grupo de estudo. As informações sobre a evolução das pacientes e dos $\mathrm{RN}$ foram recuperadas dos respectivos prontuários e coletadas em ficha especificamente planejada para esse fim, contendo todos os dados a serem avaliados na análise estatística. Posteriormente os dados foram inseridos em programa de computador Epi- Info 5.0.

Os resultados foram avaliados da PMMF, do último teste realizado em cada gestante, dentro dos três dias que antecederam o parto. Para cada registro de PMMF, realizou-se o cálculo dos valores da sensibilidade, especificidade e valores preditivo positivo (VPP) e negativo (VPN) em relação às variáveis perinatais. O mesmo procedimento foi utilizado para as demais variáveis de controle.

Por último, foram definidos três pontos de corte em relação ao tempo decorrido até obtenção dos sete movimentos: 30, 40 e 60 minutos e, para cada um, foram calculados os valores da sensibilidade, especificidade e preditivo positivo e negativo em relação às variáveis neonatais.

\section{Resultados}

A média de idade por ocasião do parto no grupo estudado foi de 31,2 anos (14-46), com número médio de gestações de 3,6 . O número de partos prévios foi de 2,1 e o de abortos de 0,6, nas pacientes não-primigestas. A média de filhos vivos, excluindo as nuliparas, foi de 1,7. Estas gestantes iniciaram o pré-natal em torno da $20^{a}$ semana e tiveram, em média, nove consultas (Tabela 1).

Foi necessário o uso de insulina para o controle metabólico em 138 das gestantes $165 \%$ das pacientes do programa de assistência). O controle foi considerado de boa qualidade em cerca de $80 \%$ do total de casos. Apenas cinco por cento das diabéticas tiveram controle ruim.

As complicações clínicas e obstétricas mais freqüentes foram, em ordem crescente, o trabalho de parto prematuro $(9 \%)$, poliidrâmnio $(11,4 \%)$, infecção do trato urinário $(24 \%)$ e a hipertensão arterial $(36,3 \%)$. Apenas $46 \%$ das hipertensas necessitaram de vasodilatador para controle da doença.
Tabela 1 - Características demográficas da população estudada. Médias, desvio padrão e faixa de variação.

\begin{tabular}{lccc}
\hline Característica & Média & Desvio padrão & Variação \\
\hline Idade & 31,2 & 6,3 & $14-46$ \\
№ gestações & 3,6 & 2,1 & $1-14$ \\
Paridade * $^{*}$ & 2,1 & 1,6 & $0-10$ \\
Aborto* $^{*}$ & 0,6 & 1,0 & $1-7$ \\
Filhos vivos ** $^{*}$ & 1,7 & 1,5 & $0-7$ \\
№ consultas & 9,1 & 3,6 & $1-19$ \\
Início do pré-natal (semanas) & 19,5 & 7,9 & $6-38$ \\
\hline
\end{tabular}

${ }^{*}$ Excluindo primigestas

${ }^{* *}$ Excluindo nulíparas

O parto foi resolvido por cesárea em 150 das 209 mulheres $(71,7 \%)$. Destas, a indicação mais freqüente foi o antecedente de cesárea $33,3 \%$, seguida do SFI $(24,7 \%)$. A idade gestacional dos RN variou entre 32 e 41 semanas, com média de 38 semanas. O peso médio foi de 3.374 gramas (com extremos de 940 e 5.110 gramas). Aproximadamente $60 \%$ dos RN tiveram peso considerado adequado para idade gestacional, $37 \%$, foram considerados grandes e $3,7 \%$ pequenos para idade gestacional.

Em relação à evolução desses RN, observamos que $59,3 \%$ não apresentaram nenhum tipo de complicação. Em 35,8\% houve hipoglicemia e em $11 \%$ sindrome de desconforto respiratório. Onze dos 209 RN tiveram diagnóstico neonatal de anóxia, embora 27 tivessem nascido com Apgar inferior a sete ao primeiro minuto e apenas seis deles tivessem Apgar de quinto minuto menor que sete. Cinco dos 209 RN faleceram no período periparto (Tabela 2).

Tabela 2 - Patologias neonatais dos recém-nascidos das pacientes diabéticas $(n=209)$.

\begin{tabular}{lrr}
\hline Patologia & $\mathbf{n}$ & $\mathbf{\%}$ \\
\hline Ausente & 124 & 59,3 \\
SDR & 23 & 11,0 \\
Hipoglicemia & 75 & 35,8 \\
Hipocalcemia & 8 & 3,8 \\
Malformação & 5 & 2,3 \\
Anóxia & 11 & 5,2 \\
Óbitos & 5 & 2,3 \\
Apgar $1^{\circ}$ minuto $<7$ & 27 & 12,9 \\
Apgar $5^{\circ}$ minuto $<7$ & 6 & 2,8 \\
IG <37 sem & 37 & 17,7 \\
\hline
\end{tabular}

SDR = Síndrome do desconforto respiratório 
Em 195 dos 209 casos (93\%) a contagem de sete movimentos foi alcançada antes de uma hora. Houve, portanto, 14 casos com suspeita de SF de acordo com a PMMF. Cento e noventa e quatro registros ou $93 \%$ foram realizados no dia ou na véspera do parto.

Dentre as 195 gestantes que registraram os sete movimentos fetais em uma hora, aproximadamente $85 \%$ conseguiram esse registro antes da primeira meia hora e mais de $95 \%$ antes dos 40 minutos (Tabela 3). Usando 40 minutos como ponto de corte, elevaram-se os valores da sensibilidade para Apgar de primeiro e de quinto minutos assim como de SFI, sem alterar o da anóxia neonatal, mas ao mesmo tempo houve perda de especificidade e do valor preditivo positivo, sem grandes mudanças no valor preditivo negativo do teste. Os valores da especificidade e preditivo negativo da PMMF foram sempre elevados e em geral acima de $90 \%$ para todas as variáveis. A sensibilidade e VPP apresentaram variações amplas, mas a sensibilidade para anóxia neonatal foi próxima de $50 \%$ (Tabela 4).

Tabela 3 - Distribuição dos resultados da PMMF, de acordo com o tempo decorrido até a anotação do sétimo movimento.

\begin{tabular}{lrr}
\hline Tempo (minutos) & $\mathbf{n}$ & $\%$ \\
\hline $0-10$ & 43 & 22,0 \\
$11-20$ & 94 & 48,2 \\
$21-30$ & 31 & 15,8 \\
$31-40$ & 18 & 9,2 \\
$41-50$ & 5 & 2,5 \\
$51-60$ & 4 & 2,0 \\
Total & 195 & 100 \\
\hline
\end{tabular}

Tabela 4 - Sensibilidade (SEN), especificidade (ESP) e valores preditivos positivo (VPP) e negativo (VPN) da PMMF em relação a indicadores de SFI, para dois pontos de corte: 60 e 40 minutos.

\begin{tabular}{lcrrrrr}
\hline & \multicolumn{2}{c}{ Apgar 5'<7 } & \multicolumn{2}{c}{ SFI } & \multicolumn{2}{c}{ A. Neonatal } \\
Tempo & $\mathbf{6 0}$ & $\mathbf{4 0}$ & $\mathbf{6 0} \mathbf{0}^{\prime}$ & $\mathbf{4 0}$ & $\mathbf{6 0}$ & $\mathbf{4 0}$ \\
\hline SEN & 23,3 & 33,3 & 28,5 & 39,2 & 45,4 & 45,4 \\
ESP & 94,0 & 89,6 & 97,4 & 90,9 & 95,4 & 90,4 \\
VPP & 14,2 & 8,3 & 80,0 & 61,1 & 35,7 & 20,8 \\
VPN & 97,9 & 97,8 & 78,9 & 80,4 & 96,9 & 96,7
\end{tabular}

Apgar 5' - Apgar 5o minuto

SFI - sofrimento fetal intraparto

A. neonatal - anóxia neonatal

Os diferentes indicadores da acurácia do teste tiveram uma melhora muito leve quando avaliado apenas no grupo de diabéticas com hipertensão arterial (Tabela 5).
Tabela 5 - Sensibilidade (SEN), especificidade (ESP) e valores preditivos positivo (VPP) e negativo (VPN) da PMMF em predizer Apgar de 10 minuto $<7$ em diabéticas com hipertensão arterial (HA) e no grupo total.

\begin{tabular}{lllll}
\hline & SEN & ESP & VPP & VPN \\
\hline HA (77) & 22,2 & 95,5 & 40,0 & 90,2 \\
Todas (209) & 18,5 & 95,0 & 35,7 & 88,7 \\
\hline
\end{tabular}

\section{Discussão}

A população de gestantes diabéticas estudada apresentou elevada proporção de patologias durante a gestação. Apenas 38\% de nossas pacientes apresentaram evolução clínica sem alterações.

Entre os RNs, encontramos elevada proporção de prematuros, de casos com depressão neonatal e anóxia. Estas duas últimas condições foram muito mais freqüentes entre nossas pacientes do que nas casuísticas citadas internacionalmente ${ }^{3,15}$.

Nossos resultados mostram que baixa proporção das gestantes diabéticas apresenta registro da PMMF alterada. Por este método, o concepto estaria comprometido em apenas uma de cada quinze grávidas com diabetes durante a gestação.

Por se tratar de método clínico de fácil execução e de baixo custo operacional, é válido nos determos na avaliação da performance do exame PMMF. Este teste apresentou baixa sensibilidade em relação ao índice de Apgar de primeiro e quinto minuto e em relação ao SFI, o que não é surpreendente, já que nenhum teste é capaz de predizer o que acontecerá durante o parto, ainda que seja por cesárea. Por isso mesmo, é encorajador que a PMMF tenha apresentado sensibilidade próxima a 50\% para predizer anóxia neonatal, que é seguramente o resultado que mais interessa evitar. Nossos resultados mostraram, ainda, que 60 minutos é o intervalo apropriado de observação, já que ao fazer o corte para o sétimo movimento aos 40 minutos, observou-se apenas ligeiro aumento de sensibilidade mas com perdas importantes na especificidade e, sobretudo, no valor preditivo positivo.

A PMMF apresentou valores de especificidade sempre superiores a $94 \%$ e teve baixo VPP em relação ao índice de Apgar de quinto minuto, valores maiores em relação ao índice de Apgar de primeiro minuto e anóxia neonatal e valor excepcionalmente bom em relação ao SFI.

Dados como estes justificam o emprego da PMMF em gestantes diabéticas e reforçam a idéia de que este seria um método adequado no 
seguimento, já que apresentou valores razoáveis de predição das condições fetais em gestantes de risco que incluem diabéticas. Estes resultados encontram suporte na literatura ${ }^{16}$.

A característica mais importante da PMMF é sua elevada especificidade e VPN quando se considera a população geral de gestantes que realiza o registro ${ }^{10}$. Estes resultados foram também observados, neste estudo, entre as diabéticas confirmando desta forma os dados que indicam ser a taxa de MF em gestantes diabéticas, com mais de 32 semanas, semelhante à das grávidas nãodiabéticas. Desta forma, é possivel admitir que a PMMF seja um método de avaliação fetal tão útil nas diabéticas quanto na população geral ${ }^{17}$.

A diminuição subjetiva dos movimentos fetais ao final da gestação nas diabéticas reflete um sinal de alerta importante quanto ao bem-estar fetal. A incidência de padrões alarmantes de movimentação fetal é duas vezes maior entre as gestantes portadoras de complicações anteparto, incluindo o diabetes, que entre as nãoportadoras ${ }^{18}$. Por este motivo, a percepção materna dos movimentos fetais tem sido, sistematicamente, recomendada em gestantes diabéticas de serviços conceituados de assistência prénatal ${ }^{19}$.

A avaliação da PMMF pela gestante neste trabalho permite perceber que as diabéticas desta série foram capazes de sentir, registrar e valorizar os movimentos fetais. Díaz et al. ${ }^{14}$ já haviam demonstrado ser possivel a realização de registro da PMMF em população de menor nível sócioeconômico, como é a que é atendida na UNICAMP. Do ponto de vista estatístico, este método poderia ser considerado excelente para aplicação populacional, ou seja, como exame de rastreamento $^{14}$

A melhor correlação com resultados perinatais desfavoráveis nas pacientes com hipertensão do que naquelas sem esta alteração vascular, apesar de não ter significação estatística, está de acordo com os resultados de Landon et al. ${ }^{20}$ que chega a recomendar que a avaliação da vitalidade fetal é necessária apenas em diabetes com esta complicação. A mínima diferença entre gestantes diabéticas hipertensas e sem esta complicação, em nossos resultados, não justifica esta recomendação.

Entre as pacientes bem controladas, do ponto de vista metabólico, e nas de termo, houve tendência a menor correlação das alterações dos exames com resultados perinatais o que encontra suporte na literatura ${ }^{21}$.

Os resultados obtidos nos permitem recomendar a utilização sistemática da PMMF em gestantes diabéticas, a partir da $32^{\text {a }}$ semana de gestação, reservando outros exames mais onerosos aos casos em que este teste estiver alterado. A alta especificidade do VPN do teste dá suficiente segurança, sem precisar de outros exames mais complexos, talvez com exceção de pacientes com complicações vasculares mais graves.

\section{SUMMARY}

Purpose: to evaluate the accuracy of maternal perception of fetal movements (MPFM) in diabetic pregnant women, using Apgar score at the 1st and 5th min of life, intrapartum fetal distress and neonatal hypoxia as parameters.

Methods: two hundred and nine diabetic women evaluated at the High Risk Prenatal Care Clinic of the Women's Hospital (CAISM) were analyzed retrospectively between June 1988 and May 1996. All patients had MPFM records within three days before delivery, fetal heart rate recordings during labor, gestational age greater than 30 weeks and a complete neonatal evaluation. MPFM was classified as normal if seven movements were recorded in $60 \mathrm{~min}$.

Results: the sensitivity of the test was 23 and 29\% for Apgar score $<7$ at the 5th min and intrapartum fetal distress, respectively, and close to $50 \%$ for neonatal hypoxia (45.5\%). Specificity was near 95\% for the three standards, and the negative predictive value (NPV) was $80 \%$ for fetal distress, increasing to 97 and $98 \%$ for Apgar $>7$ at 5 min and neonatal hypoxia.

Conclusions: MPFM is a useful test to identify diabetic women needing fetal evaluation with more complex techniques, given the high NPV, that indicates the capacity to separate the cases where the fetus is in good condition.

KEY WORDS: Diabetes in pregnancy. Fetal distress. Perinatal mortality. Diabetes mellitus.

\section{Referências}

1. Kjos SL, Leung A, Henry O, Victor MR, Paul RH, Medearis AL. Antepartum surveillance in diabetic pregnancies: predictors of fetal distress in labor. Am J Obstet Gynecol 1995; 173:1532-9.

2. Miller JM, Horger EO. Antepartum heart rate testing in diabetic pregnancy. J Reprod Med 1985; 30:515-8.

3. Kitzmiller JL, Cloherty JP, Younger MD, Tabatabaii A, Rothchild SB, Sosenko I et al. Diabetic pregnancy and perinatal morbility. Am J Obstet Gynecol 1978; 131:560-80. 
4. Olofsson P, Liedholm H, Sartor G, Sjöberg NO, Svenningsen N, Ursing D. Diabetes and pregnancy; a 21 year Swedish material. Acta Obstet Gynecol Scand Suppl 1984;122:3-62.

5. Pereira BG. Avaliação de um protocolo de assistência à grávida diabética. [Dissertação]. Campinas, Brasil: UNICAMP; 1992.

6. Lima GR, Silva HF, Grohman GS, Lopes RC, Fernandes PC, Viscomi FA et al. O. Diabetes e gravidez: estudo terapêutico de vinte casos. J Bras Ginecol 1977; 84:69-74.

7. Gettinger A, Roberts AB, Campbel S. Comparison between subjective and ultrasound assessments of fetal movement. Br Med J 1978; 2:88-90.

8. Hertogs K, Roberts AB, Cooper D, Griffin D, Campbel $\mathrm{S}$. Maternal perception of fetal motor activity. $\mathrm{Br}$ Med J 1979; 2:1183-5.

9. Faúndes A, Parpinelli MA, Pereira BG. Avaliação clínica fetal. In: Neme B, editor. Obstetrícia Básica. $1^{a}$ ed. São Paulo: Sarvier; 1994. p. 690-8.

10.Faúndes A, Pereira BG, Silva EMAF, Bacha AM, Pinotti JA. O papel da percepção materna de movimentos fetais na avaliação da vitalidade fetal. Ginecol Obstet Bras 1988; 11:92-7.

11.Pereira BG, Faúndes A. Características prégestacionais de gestantes diabéticas atendidas no Centro de Atenção Integral à Saúde da Mulher (CAISM) da Unicamp. Rev Ginecol Obstet 1995; 6:29-35.

12. Capurro H, Konichevizky S, Fonseca O, CaldeyroBarcia R. A simplified method for diagnosis of gestational age in the newborn infant. J Pediatr 1978; 93:120-2.
13.Apgar V. A proposal for a new method of evaluation of the newborn infant. Curr Res Anesth Analg 1953; 32:260-7.

14.Díaz MM, Faúndes A, Díaz J. Avaliação da factibilidade de uso do registro da percepção materna dos movimentos fetais no último trimestre da gravidez. Ginecol Obstet Bras 1985; 8:195-8.

15.Olofsson P. Improved care in diabetic pregnancy. [Thesis]. Lund, Sweden: University Hospital; 1986.

16.Liston RM, Cohen AW, Mennuti MT, Gabbe SG. Antepartum fetal evaluation by maternal perception of fetal movement. Obstet Gynecol 1982; 60:424-6.

17.Sadovsky E, Polishuk WZ. Fetal movements in utero: nature, assessment, prognostic value, timing of delivery. Obstet Gynecol 1977; 50:49-55.

18. Rayburn WF, McKean HE. Maternal perception of fetal movement and perinatal outcome. Obstet Gynecol 1980; 56:161-4.

19.Wilczynski J, Bredorowicz G. Biophysical methods of fetal assessment in diabetic mothers- how and when? Diabetes Nutr Metab 1998; 10:32.

20.Landon M.B, Langer O, Gabbe SG, Schick C, Brustman L. Fetal surveillance in pregnancies complicated by insulin-dependent diabetes mellitus. Am J Obstet Gynecol 1992; 167:617-21.

21.Roversi GD, Gargyulo M, Nicolini U, Pedretti E, Marini A, Barbarani V, et al. A new approach to the treatment of diabetic pregnant women. Report of 479 cases seen from 1963 to 1975 . Am J Obstet Gynecol 1979; 135:567-76. 